\title{
Log-Concave Polynomials II: High-Dimensional Walks and an FPRAS for Counting Bases of a Matroid
}

\author{
Nima Anari \\ Stanford University \\ Stanford, CA, USA \\ anari@cs.stanford.edu \\ Shayan Oveis Gharan \\ University of Washington \\ Seattle, WA, USA \\ shayan@cs.washington.edu
}

\author{
Kuikui Liu \\ University of Washington \\ Seattle, WA, USA \\ liukui17@cs.washington.edu \\ Cynthia Vinzant \\ North Carolina State University \\ Raleigh, NC, USA \\ clvinzan@ncsu.edu
}

\begin{abstract}
We design an FPRAS to count the number of bases of any matroid given by an independent set oracle, and to estimate the partition function of the random cluster model of any matroid in the regime where $0<q<1$. Consequently, we can sample random spanning forests in a graph and estimate the reliability polynomial of any matroid. We also prove the thirty year old conjecture of Mihail and Vazirani that the bases exchange graph of any matroid has edge expansion at least 1 .

Our algorithm and proof build on the recent results of Dinur, Kaufman, Mass and Oppenheim who show that a high dimensional walk on a weighted simplicial complex mixes rapidly if for every link of the complex, the corresponding localized random walk on the 1-skeleton is a strong spectral expander. One of our key observations is that a weighted simplicial complex $X$ is a 0 -local spectral expander if and only if a naturally associated generating polynomial $p_{X}$ is strongly log-concave. More generally, to every pure simplicial complex with positive weights on its maximal faces, we can associate to $X$ a multiaffine homogeneous polynomial $p_{X}$ such that the eigenvalues of the localized random walks on $X$ correspond to the eigenvalues of the Hessian of derivatives of $p_{X}$.
\end{abstract}

\section{CCS CONCEPTS}

- Theory of computation $\rightarrow$ Generating random combinatorial structures; Design and analysis of algorithms; Random walks and Markov chains; • Mathematics of computing $\rightarrow$ Matroids and greedoids.

\section{KEYWORDS}

Approximating Counting, Approximate Sampling, High-Dimensional Expanders, Geometry of Polynomials

Permission to make digital or hard copies of all or part of this work for personal or classroom use is granted without fee provided that copies are not made or distributed for profit or commercial advantage and that copies bear this notice and the full citation on the first page. Copyrights for components of this work owned by others than ACM must be honored. Abstracting with credit is permitted. To copy otherwise, or republish, to post on servers or to redistribute to lists, requires prior specific permission and/or a fee. Request permissions from permissions@acm.org.

STOC '19, fune 23-26, 2019, Phoenix, AZ, USA

(C) 2019 Association for Computing Machinery.

ACM ISBN 978-1-4503-6705-9/19/06

https://doi.org/10.1145/3313276.3316385

\section{ACM Reference Format:}

Nima Anari, Kuikui Liu, Shayan Oveis Gharan, and Cynthia Vinzant. 2019. Log-Concave Polynomials II: High-Dimensional Walks and an FPRAS for Counting Bases of a Matroid. In Proceedings of the 51st Annual ACM SIGACT Symposium on the Theory of Computing (STOC '19), June 23-26, 2019, Phoenix, AZ, USA. ACM, New York, NY, USA, 12 pages. https://doi.org/10.1145/ 3313276.3316385

\section{INTRODUCTION}

Let $\mu: 2^{[n]} \rightarrow \mathbb{R}_{00}$ be a probability distribution on the subsets of the set $[n]=\{1,2, \ldots, n\}$. We assign a multiaffine polynomial with variables $x_{1}, \ldots, x_{n}$ to $\mu$,

$$
g_{\mu}(x)=\sum_{S \subseteq[n]} \mu(S) \cdot \prod_{i \in S} x_{i} .
$$

The polynomial $g_{\mu}$ is also known as the generating polynomial of $\mu$. A polynomial $p \in \mathbb{R}\left[x_{1}, \ldots, x_{n}\right]$ is $d$-homogeneous if every monomial of $p$ has degree $d$. We say $\mu$ is $d$-homogeneous if the polynomial $g_{\mu}$ is $d$-homogeneous, meaning that $|S|=d$ for any $S$ with $\mu(S)>0$.

A polynomial $p \in \mathbb{R}\left[x_{1}, \ldots, x_{n}\right]$ with nonnegative coefficients is $\log$-concave on a subset $K \subseteq \mathbb{R}_{\geqslant 0}^{n}$ if $\log p$ is a concave function at any point in $K$, or equivalently, its Hessian $\nabla^{2} \log p$ is negative semidefinite on $K$. We say a polynomial $p$ is strongly log-concave on $K$ if for any $k \geqslant 0$, and any sequence of integers $1 \leqslant i_{1}, \ldots, i_{k} \leqslant n$,

$$
\left(\partial_{i_{1}} \cdots \partial_{i_{k}} p\right)\left(x_{1}, \ldots, x_{n}\right)
$$

is log-concave on $K$. In this paper, for convenience and clarity, we only work with (strong) log-concavity with respect to the allones vector, $\mathbb{1}$. So, unless otherwise specified, $K=\{\mathbb{1}\}$ in the above definition. We say the distribution $\mu$ is strongly log-concave at $\mathbb{1}$ if $g_{\mu}$ is strongly log-concave at $\mathbb{1}$. The notion of strong log-concavity was first introduced by Gurvits [Gur09; Gur10] to study approximation algorithms for mixed volume and multivariate generalizations of Newton's inequalities.

In this paper, we show that the "natural" Monte Carlo Markov Chain (MCMC) method on the support of a $d$-homogeneous strongly log-concave distribution $\mu: 2^{[n]} \rightarrow \mathbb{R}_{\geqslant 0}$ mixes rapidly. This chain can be used to generate random samples from a distribution arbitrarily close to $\mu$.

The chain $\mathcal{M}_{\mu}$ is defined as follows. We take the state space of $\mathcal{M}_{\mu}$ to be the support of $\mu$, namely $\operatorname{supp}(\mu)=\{S \subseteq[n] \mid \mu(S) \neq 0\}$. 
For $\tau \in \operatorname{supp}(\mu)$, first we drop an element $i \in \tau$, chosen uniformly at random from $\tau$. Then, among all sets $\sigma \supset \tau \backslash\{i\}$ in the support of $\mu$ we choose one with probability proportional to $\mu(\sigma)$.

It is easy to see that $\mathcal{M}_{\mu}$ is reversible with stationary distribution $\mu$. Furthermore, assuming $g_{\mu}$ is strongly log-concave, we will see that $\mathcal{M}_{\mu}$ is irreducible. We prove that this chain mixes rapidly. More formally, for a state $\tau$ of the Markov chain $\mathcal{M}$, and $\epsilon>0$, the total variation mixing time of $\mathcal{M}$ started at $\tau$ with transition probability matrix $P$ and stationary distribution $\pi$ is defined as follows:

$$
t_{\tau}(\epsilon)=\min \left\{t \in \mathbb{Z}_{\geqslant 0} \mid\left\|P^{t}(\tau, \cdot)-\pi\right\|_{1} \leqslant \epsilon\right\},
$$

where $P^{t}(\tau, \cdot)$ is the distribution of the chain started at $\tau$ at time $t$.

The following theorem is the main result of this paper.

Theorem 1.1. Let $\mu: 2^{[n]} \rightarrow \mathbb{R}_{\geqslant 0}$ be a d-homogeneous strongly log-concave probability distribution. If $P_{\mu}$ denotes the transition probability matrix of $\mathcal{M}_{\mu}$ and $X(k)$ denotes the collection of size- $k$ subsets of $[n]$ which are contained in some element of $\operatorname{supp}(\mu)$, then for every $0 \leqslant k \leqslant d-1, P_{\mu}$ has at most $|X(k)| \leqslant\left(\begin{array}{l}n \\ k\end{array}\right)$ eigenvalues of value $>1-\frac{k+1}{d}$. In particular, $\mathcal{M}_{\mu}$ has spectral gap at least $1 / d$, and if $\tau$ is in the support of $\mu$ and $0<\epsilon<1$, the total variation mixing time of the Markov chain $\mathcal{M}_{\mu}$ started at $\tau$ is at most

$$
t_{\tau}(\epsilon) \leqslant d \log \left(\frac{1}{\epsilon \mu(\tau)}\right) .
$$

To state the key corollaries of this theorem, we will need the following definition.

Definition 1.2. Given a domain set $\Omega$, which is compactly represented by, say, a membership oracle, and a nonnegative weight function $w: \Omega \rightarrow \mathbb{R}_{\geqslant 0}$, a fully polynomial-time randomized approximation scheme (FPRAS) for computing the partition function $Z=\sum_{x \in \Omega} w(x)$ is a randomized algorithm that, given an error parameter $0<\epsilon<1$ and error probability $0<\delta<1$, returns a number $\tilde{Z}$ such that $\operatorname{Prob}[(1-\epsilon) Z \leqslant \tilde{Z} \leqslant(1+\epsilon) Z] \geqslant 1-\delta$. The algorithm is required to run in time polynomial in the problem input size, $1 / \epsilon$, and $\log (1 / \delta)$.

Equipped with this definition, we can now concisely state the main applications of Theorem 1.1. Theorem 1.1 gives us an algorithm to efficiently sample from a distribution which approximates $\mu$ closely in total variation distance. By the equivalence between approximate counting and approximate sampling for self-reducible problems [JVV86], this gives an FPRAS for each of the following:

(1) approximately counting the bases of a matroid, and

(2) estimating the partition function of the random cluster model for a new range of parameter values

For real linear matroids, we also give an algorithm for estimating the partition function of a generalized version of a $k$-determinantal point process. Note that these problems are all instantiations of the following: estimate the partition function of some efficiently computable nonnegative weights on bases of a matroid. Furthermore, as the restriction and contraction of a matroid by a subset of the ground set are both (smaller) matroids, problems of this form are indeed self-reducible. In the following sections we discuss these applications in greater depth.

\subsection{Counting Problems on Matroids}

Let $M=([n], \mathcal{I})$ be an arbitrary matroid on $n$ elements (see Section 2.3) of rank $r$. Let $\mu$ be the uniform distribution on the bases of the matroid $M$. It follows that $\mu$ is $r$-homogeneous. Using the HodgeRiemann relation proved by Adiprasito, Huh, and Katz [AHK18], a subset of the authors proved [AOV18] that for any matroid $M, \mu$ is strongly log-concave. ${ }^{1}$ This implies that the chain $\mathcal{M}_{\mu}$ converges rapidly to stationary distribution. This gives the first polynomial time algorithm to generate an approximately uniformly random base of a matroid. Note that to run $\mathcal{M}_{\mu}$ we only need an oracle to test whether a given set $S \subseteq[n]$ is an independent set of $M$. Therefore, with only polynomially many queries (in $n, r, \log (1 / \epsilon)$ ) we can generate a random base of $M$.

Corollary 1.3. For any matroid $M=([n], \mathcal{I})$ of rank $r$, any basis $B$ of $M$ and $0<\epsilon<1$, the mixing time of the Markov chain $\mathcal{M}_{\mu}$ starting at $B$ is at most

$$
t_{B}(\epsilon) \leqslant r \log \left(n^{r} / \epsilon\right) \leqslant r^{2} \log (n / \epsilon) .
$$

To prove this we simply used the fact that a matroid of rank $r$ on $n$ elements has at most $\left(\begin{array}{l}n \\ r\end{array}\right) \leqslant n^{r}$ bases. There are several immediate consequences of the above corollary. Firstly, by equivalence of approximate counting and approximate sampling for self-reducible problems [JVV86] we can approximately count the number of bases of any matroid given by an independent set oracle up to a $1+\epsilon$ multiplicative error in polynomial time.

Corollary 1.4. There is a randomized algorithm that for any matroid $M$ on $n$ elements with rank $r$ given by an independent set oracle, and any $0<\epsilon<1$, counts the number of bases of $M$ up to a multiplicative factor of $1 \pm \epsilon$ with probability at least $1-\delta$ in time polynomial in $n, r, 1 / \epsilon, \log (1 / \delta)$.

As an immediate corollary for any $1 \leqslant k \leqslant r$ we can approximately count the number of independent sets of $M$ of size $k$. This is because if we truncate $M$ to independent sets of size at most $k$ it remains a matroid. As a consequence we can generate approximately uniformly random forests in a given graph, and compute the reliability polynomial

$$
C_{M}(p)=\sum_{S \subseteq[n]: \operatorname{rank}(S)=r}(1-p)^{|S|} p^{n-|S|}
$$

for any matroid and $0 \leqslant p \leqslant 1$, all in polynomial time. Note this latter fact follows from the ability to approximately count the number of independent sets of a fixed size, as the complements of rank- $r$ subsets $S \subseteq[n]$ are precisely the independent sets of the dual of $M$. Prior to our work, we could only compute the reliability polynomial for graphic matroids due to a recent work of Guo and Jerrum [GJ18a].

One can associate a graph $G_{M}$ to any matroid $M$, called the bases exchange graph. This graph has a vertex for every basis of $M$ and two bases $B, B^{\prime}$ are connected by an edge if $\left|B \Delta B^{\prime}\right|=2$. It follows by the bases exchange property of matroids that this graph

\footnotetext{
${ }^{1}$ Indeed, in [AOV18], it is shown that $g_{\mu}$ satisfies a seemingly stronger property known as "complete log-concavity", namely that $\partial_{v_{1}} \cdots \partial_{v_{k}} g_{M}$ is log-concave (at $\mathbb{1}$ ) for any sequence of directional derivatives $\partial_{v_{1}} \cdots \partial_{v_{k}}$ with nonnegative directions $v_{1}, \ldots, v_{k} \in \mathbb{R}_{\geqslant 0}^{n}$. We will prove in a future companion paper that complete logconcavity is equivalent to strong log-concavity.
} 
is connected. For an unweighted graph $G=(V, E)$, the expansion of a set $S \subset V$ and the graph $G$ are defined as

$$
\mathrm{h}(S)=\frac{|E(S, \bar{S})|}{|S|} \quad \text { and } \mathrm{h}(G)=\min _{S:|S| \leqslant|\bar{S}|} \mathrm{h}(S) .
$$

Mihail and Vazirani [MV89] conjectured that the bases exchange graph has expansion at least one, i.e., that $\mathrm{h}\left(G_{M}\right) \geqslant 1$, for any matroid $M$. It turns out that the bases exchange graph is closely related to the Markov chain $\mathcal{M}_{\mu}$. The following theorem is an immediate consequence of the above corollary.

Theorem 1.5. For any matroid $M$, the expansion of the bases exchange graph is at least $1, \mathrm{~h}\left(G_{M}\right) \geqslant 1$.

\subsection{The Random Cluster Model}

Another application of this theory is estimating the partition function of the random cluster model. For a matroid $M=([n], \mathcal{I})$ of rank $r$ and parameters $p, q$, the partition function of the random cluster model from statistical mechanics due to Fortuin and Kasteleyn [For71; FK72; For72a; For72b] is the following polynomial function associated to $M$,

$$
Z_{M}(p, q)=\sum_{S \subseteq[n]} q^{r+1-\operatorname{rank}(S)} p^{|S|}
$$

where $\operatorname{rank}(S)$ is the size of the largest independent set contained in $S$. We note that typically one scales each term by $(1-p)^{n-|S|}$ but up to a normalization factor (and change of variables) the two polynomials are equivalent. We refer interested readers to a recent book of Grimmett [Gri09] for further information. Typically, one considers the special case where $M$ is a graphic matroid, in which case the exponent of $q$ is simply the number of connected components of $S$. To the best of our knowledge, prior to this work, one could only compute $Z_{M}$ when $q=2$ because of the close connection to the Ising model [JS93; GJ17]. Our next result is a polynomial time algorithm that estimates $Z_{M}(p, q)$ for any $0<q \leqslant 1$ and $p \geqslant 0$.

Theorem 1.6. For a matroid $M$ with rank function rank : $2^{[n]} \rightarrow$ $\mathbb{Z}_{\geqslant 0}$, parameter $0<q \leqslant 1$ and choice of "external field" $\lambda=$ $\left(\lambda_{1}, \ldots, \lambda_{n}\right) \in \mathbb{R}_{>0}^{n}$, the polynomial

$$
f_{M, k, q}\left(x_{1}, \ldots, x_{n}\right)=\sum_{S \in\left(\begin{array}{c}
{[n]} \\
k
\end{array}\right)} q^{-\operatorname{rank}(S)} \prod_{i \in S} \lambda_{i} x_{i}
$$

is strongly log-concave.

Together with Theorem 1.1, this gives an FPRAS for estimating $f_{M, k, q}(\mathbb{1})$ given an independence oracle for the matroid $M$. Estimating $Z_{M}(p, q)$ then follows as

$$
Z_{M}(p, q)=q^{r+1} \sum_{k=0}^{n} p^{k} f_{M, k, q}(\mathbb{1})
$$

and each term is nonnegative. In fact, the polynomial $Z_{M}$ is closely related to the Tutte polynomial

$$
T_{M}(x, y)=\sum_{S \subseteq[n]}(x-1)^{r-\operatorname{rank}(S)}(y-1)^{|S|-\operatorname{rank}(S)} .
$$

Indeed, we can write

$$
T_{M}(x, y)=\frac{1}{(x-1)(y-1)^{r+1}} Z_{M}(y-1,(x-1)(y-1))
$$

Hence, an FPRAS for estimating $Z_{M}(p, q)$ for $p \geqslant 0$ and $0 \leqslant q \leqslant 1$ gives an FPRAS for estimating $T_{M}(x, y)$ in the region described by the inequalities $y \geqslant 1$ and $0 \leqslant(x-1)(y-1) \leqslant 1$.

\subsection{Determinantal Distributions on Real Linear Matroids}

Finally, we show that the class of homogeneous multiaffine strongly log-concave polynomials is closed under raising all coefficients to a fixed exponent less than 1.

Theorem 1.7. Let $f=\sum_{S \subseteq[n]} c_{S} \prod_{i \in S} x_{i}$ be a homogeneous degree$k$ multiaffine strongly log-concave polynomial. Then the polynomial $f_{\alpha}=\sum_{S \subseteq[n]} c_{S}^{\alpha} \prod_{i \in S} x_{i}$ is strongly log-concave for every $0 \leqslant \alpha \leqslant 1$.

We use the above theorem to design a sampling algorithm for determinantal point processes. A determinantal point process (DPP) on a set of elements $[n]$ is a probability distribution $\mu: 2^{[n]} \rightarrow \mathbb{R}_{\geqslant 0}$ identified by a positive semidefinite matrix $L \in \mathbb{R}^{n \times n}$ where for any $S \subseteq[n]$ we have

$$
\mu(S) \propto \operatorname{det}\left(L_{S}\right),
$$

where $L_{S}$ is the principal sub-matrix of $L$ indexed by the elements of $S$. Determinantal point processes are fundamental to the study of a variety of tasks in machine learning, including text summarization, image search, news threading, and diverse feature selection [see, e.g., KT12]. A $k$-determinantal point process $(k-D P P)$ is a determinantal point process conditioned on the sets $S$ having size $k$.

Given a positive semidefinite matrix $L$, let $\mu$ be the corresponding $k$-DPP. We have

$$
g_{\mu}(x) \propto \sum_{S \in\left(\begin{array}{c}
{[n]} \\
k
\end{array}\right)} \operatorname{det}\left(L_{S}\right) \cdot \prod_{i \in S} x_{i} .
$$

It turns out that the above polynomial is real stable and so it is strongly log-concave over $\mathbb{R}_{\geqslant 0}^{n}$ [see, e.g., AOR16]. Anari, Oveis Gharan, and Rezaei [AOR16] show that a natural Markov chain with the Metropolis rule mixes rapidly and generates a random sample of $\mu$. The above theorem immediately implies the following log-concavity result.

Corollary 1.8. For every positive semidefinite matrix $L \succcurlyeq 0$ and exponent $0 \leqslant \alpha \leqslant 1$, the polynomial

$$
\sum_{S \in\left(\begin{array}{c}
{[n]} \\
k
\end{array}\right)} \operatorname{det}\left(L_{S}\right)^{\alpha} \prod_{i \in S} x_{i}
$$

is strongly log-concave.

It follows from Theorem 1.1 that for any $0 \leqslant \alpha \leqslant 1$ we can generate samples from a "smoothed" $k$-DPP distribution, where for any set $S, \mathbb{P}[S] \propto \operatorname{det}\left(L_{S}\right)^{\alpha}$, in polynomial time. The weights $\operatorname{det}\left(L_{S}\right)^{\alpha}$ may be thought of as a way to interpolate between two extremes for selecting diverse data points.

We also note that for $\alpha=1 / 2$, it is known that Corollary 1.8 follows from the Brunn-Minkowski theorem applied to appropriately defined zonotopes. For $\alpha=0$ when the $k$-DPP has full support, and for $\alpha=1$ as mentioned earlier, the above polynomial is actually real stable, and hence strongly log-concave. Theorem 1.7 gives a unified proof that all of these polynomials are strongly log-concave. 


\subsection{Related Works}

There is a long line of work on designing approximation algorithms to count the bases of a matroid. Most of these works focus on expansion properties of bases exchange graph. Feder and Mihail [FM92] showed that for a special class of matroids known as balanced matroids [MS91; FM92], the bases exchange graph has expansion at least 1 . A matroid $M$ is balanced if for any minor of $M$ (including $M$ itself), the uniform distribution over its bases satisfies the pairwise negative correlation property. Many of the extensive results in this area [Gam99; JS02; Jer+04; Jer06; Clo10; CTY15; AOR16] only study approximation algorithms for this limited class of matroids, and not much is known beyond the class of balanced matroids. Unfortunately, many interesting matroids are not balanced. An important example is the matroid of all acyclic subsets of edges of a graph $G=(V, E)$ of size at most $k$ (for some $k<|V|-1$ ) [FM92].

There has been other approaches for approximately counting bases. Guo and Jerrum [GJ18b] used the popping method to approximately count bases of bicircular matroids. Barvinok and Samorodnitsky [BS07] designed a randomized algorithm that gives, roughly, a $\log (n)^{r}$ approximation factor to the number of bases of a given matroid with $n$ elements and rank $r$. In [AOV18], a subset of the authors gave a deterministic $e^{r}$ approximation to the number of bases using the fact that $g_{\mu}(M)$ is log-concave over $\mathbb{R}_{\geqslant 0}^{n}$.

There is an extensive literature on hardness of exact computation and inapproximability of the Tutte polynomial and the partition function of the random cluster model. It is known that exact computation of the Tutte polynomial for a graph is \#P-hard at all points $(x, y)$ except at $(1,1),(-1,-1),(0,-1),(-1,0)$, along the hyperbola $(x-1)(y-1)=1$, and for planar graphs, along the hyperbola $(x-1)(y-1)=2$ [JVW90], [Ver91], [Wel94]. In the realm of inapproximability, it is known that even for planar graphs, there is no FPRAS to approximate the Tutte polynomial for $x>1, y<-1$ or $y>1, x<-1$ assuming NP $\neq$ RP [GJ08; GJ12b]. Furthermore, there is no FPRAS for estimating the partition function $Z_{M}$ of the random cluster model on general graphic matroids when $q>2$, nor is there an FPRAS for $Z_{M}$ at $q=2$ for general binary matroids, unless there is an FPRAS for counting independent sets in a bipartite graph [GJ12a; GJ13; GJ14].

\subsection{Independent Work}

In a closely related work, Brändén and Huh [BH18],[BH19], in a slightly different language, have independently proved the strong log-concavity of several of the polynomials that appear in this paper. In [Ana+18b] and [BH18], both groups of authors use these techniques to prove the strongest form of Mason's conjecture. In an upcoming paper, we further study structural and closure properties of (strongly) log-concave polynomials.

\subsection{Techniques}

One of our key observations is a close connection between pure simplicial complexes and multiaffine homogeneous polynomials. Specifically, if $X$ is a pure simplicial complex with positive weights on its maximal faces, we can associate with $X$ a multiaffine homogeneous polynomial $p_{X}$ such that the eigenvalues of the localized random walks on $X$ correspond to the eigenvalues of the Hessian of derivatives of $p_{X}$. Using this correspondence, one can study multi-
Table 1: Dictionary Between Simplicial Complexes and Polynomials

\begin{tabular}{|c|c|}
\hline Weighted Simplicial Complex $X$ & Multiaffine Polynomial $p_{X}$ \\
\hline Dimension- $d$ & Degree- $d$ \\
Weight of $\emptyset$ & Evaluation at $\mathbb{1}$ \\
Connectivity of Links & Indecomposability \\
Link & Differentiation \\
Local Random Walk & (Normalized) Hessian \\
\hline
\end{tabular}

affine homogeneous polynomials using techniques from simplicial complexes, and vice versa. To study the walk $\mathcal{M}_{\mu}$ corresponding to a polynomial $g_{\mu}$, we analyze the simplicial complex corresponding to $g_{\mu}$. To do this, we leverage recent developments in the area of high-dimensional expanders, which we discuss below.

Given a simplicial complex $X$ (see Section 2.4) and an ordering of its vertices, one can associate a high dimensional Laplacian matrix to the $k$-dimensional faces of $X$. These matrices generalize the classical graph Laplacian and there has been extensive research to study their eigenvalues [see Lub17, and the references therein]. A method known as Garland's method [Gar73] relates the eigenvalues of graph Laplacians of 1-skeletons of links of $X$ to eigenvalues of high dimensional Laplacians of $X$ [see BŚ97; Opp18].

Recently, Kaufman and Mass [KM17] studied a high dimensional walk on a simplicial complex, which is closely related to the walk $\mathcal{M}_{\mu}$ that we defined above (see Section 3). Their goal is to argue that, similar to classical expander graphs, high dimensional walks mix rapidly on a high dimensional expander. Their bounds were improved in a work of Dinur and Kaufman [DK17], who showed that if all nontrivial eigenvalues of the simple random walk matrix on all 1-skeletons of links of $X$ have absolute value at most $\lambda$, then the high dimensional walk on $k$-faces of $X$ has spectral gap at least $\frac{1}{k+2}-O((k+1) \lambda)$. This was further improved in a recent work of Kaufman and Oppenheim [KO18]: They showed that if all non-trivial eigenvalues of the simple random walk matrix on all 1-skeleton of links of $X$ are at most $\lambda$, then the spectral gap of the high dimensional walk is at least $\frac{1}{k+2}-(k+1) \lambda$. In other words, negative eigenvalues of the random walk matrix do not matter. One only needs positive eigenvalues to be small.

Note that in order to make the spectral gap bounds meaningful one needs $\lambda \ll \frac{1}{k^{2}}$. In other words, one needs that, except the trivial eigenvalue of 1 , all other eigenvalues are either negative or very close to 0 . Here is the place where the connection to (strong) logconcavity comes into the picture. A polynomial $p$ is log-concave at $\mathbb{1}$ if $\nabla^{2} p(\mathbb{1})$ has at most one positive eigenvalue. A polynomial $p$ is strongly log-concave if the same holds for all partial derivatives of $p$. Our main observation is that this property is equivalent to taking $\lambda=0$ in the corresponding simplicial complex. Namely, we obtain the best possible spectral gap of $\frac{1}{k+2}$ when the simplicial complex comes from a strongly log-concave polynomial.

Our approach has a close connection to the original plan of Feder and Mihail [FM92] who used the negative correlation property of balanced matroids to show that the bases exchange walk mixes rapidly. Unfortunately, most interesting matroids do not satisfy negative correlation. But it was observed [AHK18; HW16; AOV18] that all matroids satisfy a spectral negative dependence property. 
Namely, consider the uniform distribution $\mu$ over the bases of a matroid $M$, and consider the Hessian $\nabla^{2} \log \left(g_{\mu}\right)$ of the log of the generating polynomial $g_{\mu}$ at the point $x=\mathbb{1}$. Then $M$ is negatively correlated if and only if all off-diagonal entries of this matrix are non-positive, whereas $M$ being spectrally negatively correlated means that this matrix is negative semidefinite. Spectral negative correlation is precisely what one needs to bound the mixing time of the high dimensional walk on the corresponding simplicial complex.

\section{PRELIMINARIES}

First, let us establish some notational conventions. Unless otherwise specified, all logarithms are in base $e$. All vectors are assumed to be column vectors. We use $\mathbb{R}_{>0}$ and $\mathbb{R}_{\geqslant 0}$ to denote the set of positive and nonnegative real numbers, respectively, and $[n]$ to denote $\{1, \ldots, n\}$. For a vector $x \in \mathbb{R}^{n}$ and a set $S \subseteq[n]$, we let $x^{S}$ denote $\prod_{i \in S} x_{i}$.

We use $\partial_{x_{i}}$ or $\partial_{i}$ to denote the partial differential operator $\partial / \partial x_{i}$. We denote the gradient of a function or polynomial $p$ by $\nabla p$ and the Hessian of $p$ by $\nabla^{2} p$.

\subsection{Linear Algebra}

We say a matrix $A \in \mathbb{R}^{n \times n}$ is stochastic if all entries of $A$ are nonnegative and every row adds up to exactly 1 . It is well-known that the largest eigenvalue in magnitude of any stochastic matrix is 1 and with corresponding right eigenvector being the all-ones vector, $\mathbb{1}$. If the eigenvalues of a matrix $A \in \mathbb{R}^{n \times n}$ are all real, then we order them as

$$
\lambda_{n}(A) \leqslant \cdots \leqslant \lambda_{1}(A) .
$$

For an inner product $\langle\cdot, \cdot\rangle$ on $\mathbb{R}^{n}$ (not necessarily Euclidean), we call a linear operator (or matrix) $A$ self-adjoint w.r.t. $\langle\cdot, \cdot\rangle$ when $\langle u, A v\rangle=\langle A u, v\rangle$, for all $u, v \in \mathbb{R}^{n}$.

In this paper, we will often switch between different inner products. As such, we highlight the following variational characterization of eigenvalues of a linear operator that is self-adjoint with respect to an arbitrary inner product. In particular, the matrix of the linear operator need not be symmetric.

Theorem 2.1 (Courant-Fischer Theorem). Let $A: \mathbb{R}^{n} \rightarrow \mathbb{R}^{n}$ be a linear operator that is self-adjoint with respect to some inner product $\langle\cdot, \cdot\rangle$ (not necessarily Euclidean). Then

$$
\lambda_{k}(A)=\min _{U} \max _{v}\langle v, A v\rangle,
$$

where the minimum is taken over all $(n-k)$-dimensional subspaces $U \subseteq \mathbb{R}^{n}$ and the maximum is taken over all vectors $v \in U$ with $\langle v, v\rangle=1$.

We call a self-adjoint $A$ positive semidefinite (PSD) when for all $v \in \mathbb{R}^{n}$

$$
\langle v, A v\rangle \geqslant 0
$$

In this case, we write $A \succcurlyeq 0$. By Theorem 2.1, this is equivalent to $A$ having nonnegative eigenvalues. We call $A$ negative semidefinite (NSD) when the reverse inequality holds for all $v \in \mathbb{R}^{n}$, which is equivalent to $A$ having nonpositive eigenvalues. In this case, we write $A \preccurlyeq 0$. Finally, when we have strict inequalities, we say that $A$ is positive definite (or negative definite), and write $A>0$ ( or $A<0$ ). When comparing two matrices $A, B$ which are self-adjoint w.r.t. the same inner product, we write $A \preccurlyeq B$ if and only if $B-A \succcurlyeq 0$. This is the Loewner order w.r.t. to the inner product.

For the remainder of the paper, we will use $\langle\cdot, \cdot\rangle$ to denote the usual Euclidean inner product, and $\preccurlyeq$ to denote the usual Loewner order for symmetric matrices. When we switch to a new inner product, we will add appropriate subscripts to the inner product and to the Loewner order to make it clear which inner product we are working with.

For the rest of this subsection, we collect facts from linear algebra that we will need. Proofs of several lemmas and facts can be found in the full version of the paper [Ana+18a].

Theorem 2.2 (Cauchy Interlacing Theorem [HJ13, Cor. 4.3.9]). For a symmetric matrix $A \in \mathbb{R}^{n \times n}$ and vector $v \in \mathbb{R}^{n}$, the eigenvalues of $A$ interlace the eigenvalues of $A+v v^{\top}$. That is, for $B=A+v v^{\top}$,

$$
\lambda_{n}(A) \leqslant \lambda_{n}(B) \leqslant \lambda_{n-1}(A) \leqslant \cdots \leqslant \lambda_{2}(B) \leqslant \lambda_{1}(A) \leqslant \lambda_{1}(B) .
$$

The following is an immediate consequence:

Lemma 2.3. Let $A \in \mathbb{R}^{n \times n}$ be a symmetric matrix and let $P \in \mathbb{R}^{m \times n}$. If $A$ has at most one positive eigenvalue, then $P A P^{\top}$ has at most one positive eigenvalue.

Fact 2.4. Let $A \in \mathbb{R}^{n \times k}$ and $B \in \mathbb{R}^{k \times n}$ be arbitrary matrices. Then, non-zero eigenvalues of $A B$ are equal to non-zero eigenvalues of $B A$ with the same multiplicity.

Lemma 2.5. Let $A \in \mathbb{R}^{n \times n}$ be a symmetric matrix with at most one positive eigenvalue. Then, for any PSD matrix $B \in \mathbb{R}^{n \times n}, B A$ has at most one positive eigenvalue.

Lemma 2.6. Let $A \in \mathbb{R}^{n \times n}$ be a symmetric matrix with nonnegative entries and at most one positive eigenvalue, and let $w(i)=\sum_{j=1}^{n} A_{i, j}$. Then,

$$
A \preccurlyeq \frac{w w^{\top}}{\sum_{i} w(i)} .
$$

\subsection{Markov Chains and Random Walks}

For this paper, we consider a Markov chain as a triple $(\Omega, P, \pi)$ where $\Omega$ denotes the (finite) state space, $P \in \mathbb{R}_{\geqslant 0}^{\Omega \times \Omega}$ denotes the transition probability matrix and $\pi \in \mathbb{R}_{\geqslant 0}^{\Omega}$ denotes a stationary distribution of the chain (which will be unique for all chains we consider). For $\tau, \sigma \in \Omega$, we use $P(\tau, \sigma)$ to denote the corresponding entry of $P$, which is the probability of moving from $\tau$ to $\sigma$. We say a Markov chain is $\epsilon$-lazy if for any state $\tau \in \Omega, P(\tau, \tau) \geqslant \epsilon$. A chain $(\Omega, P, \pi)$ is reversible if there is a nonzero nonnegative function $f: \Omega \rightarrow \mathbb{R}_{\geqslant 0}$ such that for any pair of states $\tau, \sigma \in \Omega$,

$$
f(\tau) P(\tau, \sigma)=f(\sigma) P(\sigma, \tau) .
$$

If this condition is satisfied, then $f$ is proportional to a stationary distribution of the chain. In this paper we only work with reversible Markov chains. Note that being reversible means that the transition matrix $P$ is self-adjoint w.r.t. the following $\langle\cdot, \cdot\rangle$ defined for $\phi, \psi \in$ $\mathbb{R}^{\Omega}$ :

$$
\langle\phi, \psi\rangle=\sum_{x \in \Omega} f(x) \phi(x) \psi(x) .
$$

Reversible Markov chains can be realized as random walks on weighted graphs. Given a weighted graph $G=(V, E, w)$ where every edge $e \in E$ has weight $w(e)$, the non-lazy simple random 
walk on $G$ is the Markov chain that from any vertex $u \in V$ chooses an edge $e=\{u, v\}$ with probability proportional to $w(e)$ and jumps to $v$. We can make this walk $\epsilon$-lazy by staying at every vertex with probability $\epsilon$. It turns out that if $G$ is connected, then the walk has a unique stationary distribution where $\pi(u) \propto w(u)$, where $w(u)=\sum_{v \sim u} w(\{u, v\})$ is the weighted degree of $u$.

For any reversible Markov chain $(\Omega, P, \pi)$, the largest eigenvalue of $P$ is 1 . We let $\lambda^{*}(P)$ denote the second largest eigenvalue of $P$ in absolute value. That is, if $-1 \leqslant \lambda_{n} \leqslant \cdots \leqslant \lambda_{1}=1$ are the eigenvalues of $P$, then $\lambda^{*}(P)$ equals $\max \left\{\left|\lambda_{2}\right|,\left|\lambda_{n}\right|\right\}$.

Theorem 2.7 ([DS91, Prop 3]). For any reversible irreducible Markov chain $(\Omega, P, \pi), \epsilon>0$, and any starting state $\tau \in \Omega$,

$$
t_{\tau}(\epsilon) \leqslant \frac{1}{1-\lambda^{*}(P)} \cdot \log \left(\frac{1}{\epsilon \cdot \pi(\tau)}\right) .
$$

For our results, it will be enough to look at the second largest eigenvalue $\lambda_{2}(P)$, which we can bound using the conductance of a weighted graph. Consider a weighted graph $G=(V, E, w)$ and a subset $S \subseteq V$ of vertices. We let $\bar{S}$ denote the complement $V \backslash S$. Then the conductance of $S$, denoted by cond $(S)$, is defined as

$$
\operatorname{cond}(S)=\frac{w(E(S, \bar{S}))}{\operatorname{vol}(S)}=\frac{\sum_{e \in E(S, \bar{S})} w(e)}{\sum_{v \in S} w(v)},
$$

where $E(S, \bar{S})=\{\{u, v\} \in E \mid u \in S, v \notin S\}$ is the set of edges between $S$ and $\bar{S}, w(E(S, \bar{S}))$ is the sum of weights of these edges, and the volume $\operatorname{vol}(S)$ is the sum of the weighted degrees of the vertices in $S$. The conductance of $G$ is then

$$
\operatorname{cond}(G)=\min _{S} \operatorname{cond}(S),
$$

where the minimum is taken over subsets $\emptyset \subsetneq S \subsetneq V$ for which $\operatorname{vol}(S) \leqslant \operatorname{vol}(\bar{S})$.

We say $G$ is $d$-regular if $w(v)=d$ for all $v \in V$.

Theorem 2.8 (Cheeger's Inequalities [AM85; Alo86]). For anydregular weighted graph $G=(V, E, w)$,

$$
\frac{d-\lambda_{2}\left(A_{G}\right)}{2} \leqslant \operatorname{cond}(G) \leqslant \sqrt{2\left(d-\lambda_{2}\left(A_{G}\right)\right)},
$$

where $A_{G}$ is the weighted adjacency matrix of $G$ given by $\left(A_{G}\right)_{i j}=$ $w(\{i, j\})$.

A direct consequence of the above theorem is that if $G$ is connected, i.e. $w(E(S, \bar{S}))>0$ holds for all nonempty proper subsets $S$ of vertices, then $\lambda_{2}\left(A_{G}\right)<d$. If the matrix $A_{G}$ is stochastic, then the graph is 1-regular, which gives the following.

Corollary 2.9. If $A \in \mathbb{R}^{n \times n}$ is a stochastic matrix corresponding to a reversible Markov chain with the property that $\sum_{|\{i, j\} \cap S|=1} A_{i, j}>0$ for all subsets $\emptyset \subsetneq S \subsetneq[n]$, then $\lambda_{2}(A)<1$.

\subsection{Matroids}

A matroid $M=([n], \mathcal{I})$ is a combinatorial structure consisting of a ground set $[n]$ of elements and a nonempty collection $\mathcal{I}$ of independent subsets of $[n]$ satisfying:

i) If $S \subseteq T$ and $T \in \mathcal{I}$, then $S \in \mathcal{I}$ (hereditary property).

ii) If $S, T \in \mathcal{I}$ and $|T|>|S|$, then there exists an element $i \in T \backslash S$ such that $S \cup\{i\} \in \mathcal{I}$ (exchange axiom).
The rank, denoted by $\operatorname{rank}(S)$, of a subset $S \subset[n]$ is the size of any maximal independent set of $M$ contained in $S$. Thus, the independent sets of $M$ are precisely those subsets $S \subset[n]$ for which $\operatorname{rank}(S)=|S|$. We call $\operatorname{rank}([n])$ the $\operatorname{rank}$ of $M$, and if $M$ has $\operatorname{rank} r$, any set $S \in \mathcal{I}$ of size $r$ is called a basis of $M$.

An element $i \in[n]$ is a loop if $\{i\} \notin \mathcal{I}$, that is, $\{i\}$ is dependent. Two non-loops $i, j \in[n]$ are parallel if $\{i, j\} \notin \mathcal{I}$, that is, $\{i, j\}$ is dependent.

Definition 2.10 (Contraction). Let $M=([n], \mathcal{I})$ be a matroid and $S \in \mathcal{I}$. Then the contraction $M / S$ is the matroid with ground set $[n] \backslash S$ and independent sets $\{T \subseteq[n] \backslash S \mid T \cup S \in \mathcal{I}\}$.

We will use a key property of matroids called the matroid partition property. For any matroid $M=([n], \mathcal{I})$, the non-loops of $M$ can be partitioned into sets $S_{1}, S_{2}, \ldots, S_{k}$ for some $1 \leqslant k \leqslant n$ with the property that non-loops $j, k \in[n]$ are parallel if and only if they belong to the same set $S_{i}$. Indeed, one can check from the axioms for a matroid that being parallel defines an equivalence relation on the non-loop elements of $[n]$ and $S_{1}, \ldots, S_{k}$ are then the corresponding equivalence classes.

\subsection{Simplicial Complexes}

A simplicial complex $X$ on the ground set $[n]$ is a nonempty collection of subsets of $[n]$ that is downward closed, namely if $\tau \subset \sigma$ and $\sigma \in X$, then $\tau \in X$. The elements of $X$ are called faces/simplices, and the dimension of a face $\tau \in X$ is defined as $\operatorname{dim}(\tau)=|\tau|$. Note that for convenience and clarity of notation, our definition deviates from the standard definition of $\operatorname{dim}(\tau)=|\tau|-1$ used by topologists.

The empty set $\emptyset$ has dimension- 0 . A face of dimension 1 is a vertex of $X$ and a face of dimension 2 is called an edge. More generally, we write

$$
X(k)=\{\tau \in X \mid \operatorname{dim}(\tau)=k\}
$$

for the collection of dimension- $k$ faces, or $k$-faces/ $k$-simplices, of $X$. The dimension of $X$ is the largest $k$ for which $X(k)$ is nonempty, and we say that $X$ is pure of dimension $d$ if all maximal faces of $X$ have the dimension $d$. In this paper we will only consider pure simplicial complexes.

The link of a face $\tau \in X$ denoted by $X_{\tau}$ is the simplicial complex on $[n] \backslash \tau$ obtained by taking all faces in $X$ that contain $\tau$ and removing $\tau$ from them,

$$
X_{\tau}=\{\sigma \backslash \tau \mid \sigma \in X, \sigma \supset \tau\}
$$

Note that if $X$ is pure of dimension $d$ and $\tau \in X(k)$, then $X_{\tau}$ is pure and has dimension $(d-k)$.

For any matroid $M=([n], \mathcal{I})$ of rank $r$, the independent sets $\mathcal{I}$ form a pure $r$-dimensional simplicial complex on $[n]$ called its independence (or matroid) complex. Furthermore, for any $S \in \mathcal{I}$, the link $I_{S}$ of the independence complex consists precisely of the independent sets of the contraction $M / S$. There are many other beautiful simplicial complexes associated to matroids, but here we will be mainly interested in the independence complex.

We can equip a simplicial complex with a weight function: $w$ : $X \rightarrow \mathbb{R}_{>0}$ which assigns a positive weight to each face of $X$. We say that $w$ is balanced if for every non-maximal face $\tau \in X$ of 
dimension $k$,

$$
w(\tau)=\sum_{\sigma \in X(k+1): \sigma \supset \tau} w(\sigma)
$$

For a pure simplicial complex $X$ we can define a balanced weight function by assigning arbitrary positive weights to maximal faces and defining the weight of each lower dimensional face recursively. Indeed, if $X$ is a pure simplicial complex of dimension $d$ and $w$ is a balanced weight function, then, for any $\tau \in X(k)$,

$$
w(\tau)=(d-k) ! \sum_{\sigma \in X(d): \sigma \supset \tau} w(\sigma) .
$$

One natural choice is the function which assigns a weight of one to each maximal face, but there are many other interesting choices.

Any balanced weight function on $X$ induces a weighted graph on the vertices of $X$ as follows. The 1-skeleton of $X$ is the graph on vertices $X(1)$ with edges $X(2)$. Then, restricting $w$ to $X(1)$ and $X(2)$ determines a weighted graph, where $w(v)$ gives the weighted degree of each $v \in X(1)$. The weighted graphs coming from both $X$ and its links $X_{\tau}$ will be useful in later sections.

\subsection{Log-Concave Polynomials}

We say a polynomial $p \in \mathbb{R}\left[x_{1}, \ldots, x_{n}\right]$ is $d$-homogeneous if every monomial of $p$ has degree $d$; equivalently, $p$ is $d$-homogeneous if $p\left(\lambda x_{1}, \ldots, \lambda x_{n}\right)=\lambda^{d} p\left(x_{1}, \ldots, x_{n}\right)$ for every $\lambda \in \mathbb{R}$. For a $d$ homogeneous polynomial $p$, the following identity, known as Euler's identity, holds:

$$
d \cdot p(x)=\sum_{k=1}^{n} x_{k} \partial_{k} p(x) .
$$

Note that if $p$ is homogeneous then all directional derivatives of $p$ are also homogeneous, so one can apply this to $\partial_{i} p(x)$ and $\partial_{i} \partial_{j} p(x)$ to find that

$$
\begin{aligned}
& (d-1) \cdot(\nabla p)=\sum_{k=1}^{n} x_{k} \cdot\left(\nabla\left(\partial_{k} p\right)\right)=\left(\nabla^{2} p\right) \cdot x \\
& (d-2) \cdot\left(\nabla^{2} p\right)=\sum_{k=1}^{n} x_{k} \cdot\left(\nabla^{2}\left(\partial_{k} p\right)\right) .
\end{aligned}
$$

A polynomial $p \in \mathbb{R}\left[x_{1}, \ldots, x_{n}\right]$ with nonnegative coefficients is $\log$-concave if $\log p$ is a concave function over $\mathbb{R}_{>0}^{n}$. For simplicity we also consider the zero polynomial to be log-concave. Equivalently, $p$ is $\log$-concave if the Hessian of $\log p$

$$
\nabla^{2} \log p=\frac{p \cdot\left(\nabla^{2} p\right)-(\nabla p)(\nabla p)^{\top}}{p^{2}}
$$

is negative semidefinite at any point $x \in \mathbb{R}_{>0}^{n}$, where $\nabla p$ is the gradient of $p$. Since $(\nabla p)(\nabla p)^{\top}$ is a rank-1 matrix, by Cauchy's interlacing theorem, $p \cdot\left(\nabla^{2} p\right)$ has at most one positive eigenvalue at any $x \in \mathbb{R}_{>0}^{n}$. Since $p$ has nonnegative coefficients and $x$ has strictly positive entries, $p(x)>0$ so $\nabla^{2} \log p$ being negative semidefinite is equivalent to $\nabla^{2} p \preccurlyeq \frac{(\nabla p)(\nabla p)^{\top}}{p}$, where the right-hand side is a rank-1 positive semidefinite matrix. In particular, $\nabla^{2} p$ has at most one positive eigenvalue at $x$. In [AOV18] it is shown that for homogeneous polynomials $p$, the converse of this is also true, i.e., if $\nabla^{2} p$ has at most one positive eigenvalue at all $x>0$ then $p$ is $\log$-concave.
Proposition 2.11 ([AOV18]). A degree-d homogeneous polynomial $p \in \mathbb{R}\left[x_{1}, \ldots, x_{n}\right]$ with nonnegative coefficients is log-concave over $\mathbb{R}_{>0}^{n}$ iff $\left(\nabla^{2} p\right)(x)$ has at most one positive eigenvalue at all $x \in \mathbb{R}_{>0}^{n}$.

Remark 2.12. Whenever $p$ has degree at least 2 and nonnegative coefficients, $\left(\nabla^{2} p\right)(x)$ has at least one positive entry for any $x \in \mathbb{R}_{>0}^{n}$. Hence, one can see (via, for example, the variational characterization of eigenvalues Theorem 2.1 and using a test vector with positive entries) that $\left(\nabla^{2} p\right)(x)$ must have at least one strictly positive eigenvalue. Thus, whenever we write "at most one positive eigenvalue", we also mean it has "exactly one positive eigenvalue".

We say a polynomial $p \in \mathbb{R}\left[x_{1}, \ldots, x_{n}\right]$ is decomposable if it can be written as a sum of polynomials in disjoint subset of the variables, that is, if there exists a nonempty subset $I \subsetneq[n]$ and nonzero polynomials $g \in \mathbb{R}\left[x_{i}: i \in I\right], h \in \mathbb{R}\left[x_{i}: i \notin I\right]$ for which $f=g+h$. We call $f$ indecomposable otherwise.

Lemma 2.13. If $p \in \mathbb{R}\left[x_{1}, \ldots, x_{n}\right]$ has nonnegative coefficients, is homogeneous of degree at least 2 , and log-concave at $\mathbb{1}$, then $p$ is indecomposable.

Proof. See the full version of the paper [Ana+18a].

In order to prove several distributions of interest are strongly logconcave, we will prove an equivalent characterization of strongly log-concave polynomials.

Theorem 2.14. Let $p \in \mathbb{R}\left[x_{1}, \ldots, x_{n}\right]$ be a d-homogeneous polynomial such that:

(1) for any $0 \leqslant k \leqslant d-2$ and any $\left(i_{1}, \ldots, i_{k}\right) \in[n]^{k}, \partial_{i_{1}} \cdots \partial_{i_{k}} p$ is indecomposable, and

(2) for any $\left(i_{1}, \ldots, i_{d-2}\right) \in[n]^{d-2}$, the quadratic $\partial_{i_{1}} \ldots \partial_{i_{d-2}} p$ is either identically zero, or log-concave at $\mathbb{1}$.

Then $p$ is strongly log-concave at $\mathbb{1}$.

Note that Lemma 2.13 shows that the condition that all partial derivatives are indecomposable is necessary in order for a polynomial to be (strongly) log-concave.

\section{WALKS ON SIMPLICIAL COMPLEXES}

Consider a pure $d$-dimensional complex $X$ with a balanced weight function $w: X \rightarrow \mathbb{R}_{>0}$. We will call $(X, w)$ a weighted complex. For $1 \leqslant k<d$, we define a random walk on $X(k)$ known as the upper $k$-walk based on movement from an face in $X(k)$ to a higherdimensional face and then returning to $X(k)$. Similarly, for $1 \leqslant k<$ $d$, we define a random walk on $X(k+1)$ known as the lower $k$-walk based on movement from a face in $X(k+1)$ to a lower-dimensional face and then returning to $X(k+1)$.

To define these walks we construct a bipartite graph $G_{k}$ with one side corresponding to $X(k)$ and the other side corresponding to $X(k+1)$. We connect $\tau \in X(k)$ to $\sigma \in X(k+1)$ with an edge of weight $w(\sigma)$ iff $\tau \subset \sigma$. Now, consider the simple (weighted) random walk on $G_{k}$. Given a vertex we choose a neighbor proportional to the weight of the edge connecting the two vertices.

This is a walk on a bipartite graph and is naturally periodic. We can consider the odd steps and even steps, in order to obtain two random walks; one on $X(k)$ called $P_{k}^{\wedge}$, and the other on $X(k+1)$ called $P_{k+1}^{\vee}$, where given $\tau \in X(k)$ you take two steps of the walk in 
$G_{k}$ to transition to the next $k$-face with respect to the $P_{k}^{\wedge}$ matrix, and similarly, you take two steps in $G_{k}$ from $\sigma \in X(k+1)$ to transition with respect to $P_{k+1}^{\vee}$.

Now, let us formally write down the entries of $P_{k}^{\wedge}$ and $P_{k+1}^{\vee}$. Given a simplex $\tau \in X(k)$, first among all $k+1$ dimensional simplices $\sigma \in X(k+1)$ that contain $\tau$ we choose one proportional to $w(\sigma)$. Then, we delete one of the $|\sigma|=k+1$ elements of $\sigma$ uniformly at random to obtain a new state $\tau^{\prime}$. It follows that the probability of transition to $\tau^{\prime}$ is equal to the probability of choosing $\sigma=\tau \cup \tau^{\prime}$ in the first step, which is equal to $\frac{w\left(\tau \cup \tau^{\prime}\right)}{w(\tau)}$ since $w$ is balanced, times the probability of choosing $\tau^{\prime}$ conditioned on $\sigma=\tau \cup \tau^{\prime}$, which is $\frac{1}{k+1}$. In summary, for $1 \leqslant k<d$,

$$
P_{k}^{\wedge}\left(\tau, \tau^{\prime}\right)= \begin{cases}\frac{1}{k+1}, & \text { if } \tau=\tau^{\prime} \\ \frac{w\left(\tau \cup \tau^{\prime}\right)}{(k+1) w(\tau)}, & \text { if } \tau \cup \tau^{\prime} \in X(k+1) \\ 0, & \text { otherwise }\end{cases}
$$

Note that upper walk is not defined for $k=d$, because there is no $(d+1)$-dimensional simplex in $X$.

Analogously, given $\sigma \in X(k+1)$, first we remove a uniformly random element of $\sigma$ to obtain $\tau$. Then, among all all $k+1$ simplices $\sigma^{\prime} \in X(k+1)$ that contain $\tau$ we choose one proportional to $w\left(\sigma^{\prime}\right)$. It follows that for $1 \leqslant k<d$,

$$
P_{k+1}^{\vee}\left(\sigma, \sigma^{\prime}\right)= \begin{cases}\sum_{\tau \in X(k): \tau \subset \sigma} \frac{w(\sigma)}{(k+1) w(\tau)}, & \text { if } \sigma=\sigma^{\prime} \\ \frac{w\left(\sigma^{\prime}\right)}{(k+1) w\left(\sigma \cap \sigma^{\prime}\right)}, & \text { if } \sigma \cap \sigma^{\prime} \in X(k) \\ 0, & \text { otherwise }\end{cases}
$$

Observe the corresponding random walks are reversible with respect to the weight function $w$, i.e., for all $\tau, \tau^{\prime} \in X(k)$, we have

$$
\begin{aligned}
& w(\tau) P_{k}^{\wedge}\left(\tau, \tau^{\prime}\right)=w\left(\tau^{\prime}\right) P_{k}^{\wedge}\left(\tau^{\prime}, \tau\right) \\
& w(\tau) P_{k}^{\vee}\left(\tau, \tau^{\prime}\right)=w\left(\tau^{\prime}\right) P_{k}^{\vee}\left(\tau^{\prime}, \tau\right) .
\end{aligned}
$$

This implies that both chains have the same stationarity distribution where the probability of $\tau \in X(k)$ is proportional to $w(\tau)$.

Lemma 3.1. For any $1 \leqslant k<d, P_{k}^{\wedge}$ and $P_{k+1}^{\vee}$ are stochastic, selfadjoint w.r.t. the $w$-induced inner product, PSD, and have the same (with multiplicity) non-zero eigenvalues.

Proof Sкetch. Let $P_{k}$ be the transition probability matrix of the simple random walk on $G_{k}$. Since $G_{k}$ is bipartite and we can write

$$
P_{k}=\left[\begin{array}{cc}
0 & P_{k}^{\downarrow} \\
P_{k}^{\uparrow} & 0
\end{array}\right] \quad P_{k}^{2}=\left[\begin{array}{cc}
P_{k}^{\downarrow} P_{k}^{\uparrow} & 0 \\
0 & P_{k}^{\uparrow} P_{k}^{\downarrow}
\end{array}\right]
$$

where $P_{k}^{\downarrow} \in \mathbb{R}^{X(k+1) \times X(k)}$ and $P_{k}^{\uparrow} \in \mathbb{R}^{X(k) \times X(k+1)}$ are stochastic matrices.

The key observation is that $P_{k}^{\wedge}$ and $P_{k+1}^{\vee}$ correspond to two step walks on $G_{k}$. Indeed we can write

$$
P_{k}^{\wedge}=P_{k}^{\uparrow} P_{k}^{\downarrow} \quad P_{k+1}^{\vee}=P_{k}^{\downarrow} P_{k}^{\uparrow}
$$

Hence, they clearly have the same non-zero eigenvalues (with multiplicity), and the fact that they are self-adjoint and PSD are inherited from $P_{k}^{2}$ being self-adjoint and PSD. A detailed proof is given in the full version of the paper [Ana+18a].
Let us specifically study $P_{1}^{\wedge}$. Observe that $P_{1}^{\wedge}$ is the transition probability matrix of the simple (1/2)-lazy random walk on the weighted 1-skeleton of $X$ where the weight of each edge $e \in X(2)$ is $w(e)$. We also need to consider the non-lazy variant of this random walk, given by the transition matrix

$$
\tilde{P}_{1}^{\wedge}=2\left(P_{1}^{\wedge}-I / 2\right)
$$

Similarly, for any face $\tau \in X(k)$, we define the upper random walk on the faces of the link $X_{\tau}$. Specifically, let $P_{\tau, 1}^{\wedge}$ denote the transition matrix of the upper walk, as above, on the 1-dimensional faces of $X_{\tau}$, and

$$
\tilde{P}_{\tau, 1}^{\wedge}=2\left(P_{\tau, 1}^{\wedge}-I / 2\right)
$$

be the transition matrix for the non-lazy version.

Definition 3.2 (Local Spectral Expanders, [KO18]). For $\lambda \geqslant 0$, a pure $d$-dimensional weighted complex $(X, w)$ is a $\lambda$-local-spectralexpander if for every $0 \leqslant k<d-1$, and for every $\tau \in X(k)$, we have $\lambda_{2}\left(\tilde{P}_{\tau, 1}^{\wedge}\right) \leqslant \lambda$.

In other words, $X$ is $\lambda$-local spectral expander if the spectral gap of the natural random walk on the 1-skeleton of the link of all simplices of $X$ has a spectral gap of at least $1-\lambda$. In this section we give a somewhat simpler proof of the following special case of the main theorem of [KO18].

Theorem 3.3 ([KO18]). Let $(X, w)$ be a pured-dimensional weighted 0 -local spectral expander and let $0 \leqslant k<d$. Then, for all $-1 \leqslant i \leqslant k$, $P_{k}^{\wedge}$ has at most $|X(i)| \leqslant\left(\begin{array}{c}n \\ i\end{array}\right)$ eigenvalues of value $>1-\frac{i+1}{k+1}$, where for convenience, we set $X(-1)=\emptyset$ and $\left(\begin{array}{c}n \\ -1\end{array}\right)=0$. In particular, the second largest eigenvalue of $P_{k}^{\wedge}$ is at most $\frac{k}{k+1}$.

Remark 3.4. In other words, $P_{k}^{\wedge}$ has very few "big" eigenvalues. For example, $P_{k}^{\wedge}$ has exactly one eigenvalue strictly larger than $\frac{k}{k+1}$ corresponding to the maximum eigenvalue (which has value 1 ) and at most $n=|X(1)|$ eigenvalues strictly larger than $\frac{k-1}{k+1}$. Hence, $P_{k}^{\wedge}$ has at most $n-1$ eigenvalues between $\frac{k-1}{k+1}$ and $\frac{k}{k+1}$. Note that the significance of this theorem is that we are able to establish an estimate on all eigenvalues of $P_{k}^{\wedge}$.

For the proof, we will need the following lemma. We remark that the inner product on the space $\mathbb{R}^{X(k)}$ is given by $\langle\phi, \psi\rangle_{k}=$ $\sum_{\tau \in X(k)} w(\tau) \phi(\tau) \psi(\tau)$, and that being self-adjoint, PSD, and the Loewner order are defined w.r.t. this inner product. For clarity, we write $A \preccurlyeq_{k} B$ if $A, B \in \mathbb{R}^{X(k) \times X(k)}$ are self-adjoint w.r.t. $\langle\cdot, \cdot,\rangle_{k}$ and satisfy $\langle\phi, A \phi\rangle_{k} \leq\langle\phi, B \phi\rangle_{k}$ for all $\phi \in \mathbb{R}^{X(k)}$. We use $\preccurlyeq$ for the usual Loewner order induced by the Euclidean inner product.

Lemma 3.5. Let $(X, w)$ be a pure d-dimensional weighted 0-local spectral expander. Then for every $0 \leq k<d$, we have the following relation between the upper $k$-walk, and the lower $(k-1)$-walk: $P_{k}^{\wedge} \preccurlyeq_{k}$ $\frac{k}{k+1} P_{k}^{\vee}+\frac{1}{k+1} I$

Proof. For convenience, let $M=P_{k}^{\wedge}-\left(\frac{k}{k+1} P_{k}^{\vee}+\frac{1}{k+1} I\right)$. Fix $\eta \in X(k-1)$. We will first consider submatrices $M_{\eta}$ whose entries are given by the following:

$$
M_{\eta}(\tau, \sigma)= \begin{cases}M(\tau, \sigma), & \text { if } \tau \neq \sigma, \eta=\tau \cap \sigma \\ -\frac{1}{k+1} \cdot \frac{w(\tau)}{w(\eta)}, & \text { if } \tau=\sigma, \tau \supset \eta \\ 0, & \text { otherwise }\end{cases}
$$


Note that $M=\sum_{\eta \in X(k-1)} M_{\eta}$ and hence, it suffices to prove that $M_{\eta} \preccurlyeq_{k} 0$ for every $\eta \in X(k-1)$.

Fix $\eta \in X(k-1)$. Let $\tau, \sigma \in X(k)$ with $\tau \neq \sigma$ and $\tau \cap \sigma=\eta$. Then

$$
\begin{aligned}
M_{\tau \cap \sigma}(\tau, \sigma) & =M(\tau, \sigma) \\
& =\frac{1}{k+1}\left(\frac{w(\tau \cup \sigma)}{w(\tau)}-\frac{w(\sigma)}{w(\tau \cap \sigma)}\right) \\
& =\frac{1}{k+1}\left(\frac{w(\tau \cup \sigma) w(\tau \cap \sigma)-w(\tau) w(\sigma)}{w(\tau) w(\tau \cap \sigma)}\right)
\end{aligned}
$$

Furthermore, by definition, if $\tau \in X(k)$ with $\tau \supset \eta$, then $M_{\eta}(\tau, \tau)=$ $-\frac{1}{k+1} \cdot \frac{w(\tau)}{w(\eta)}$. A matrix calculation reveals that

$$
M_{\eta}=\frac{1}{(k+1) w(\eta)} \operatorname{diag}\left(w_{\eta}\right)^{-1} \cdot\left(w(\eta) \cdot A_{\eta}-w_{\eta} w_{\eta}^{\top}\right)
$$

where $w_{\eta}$ is the $|X(k)|$-dimensional vector whose non-zero entries are $w(\tau)$ for $\tau \supset \eta$, and $A_{\eta}$ is the symmetric $|X(k)| \times|X(k)|$ matrix whose non-zero entries are $w(\tau \cup \sigma)$ for $\tau, \sigma \in X(k)$ satisfying $\tau \cup \sigma \in X(k+1)$ and $\tau \cap \sigma=\eta$. Note that $M_{\eta} \preccurlyeq k 0$, if and only if $\operatorname{diag}\left(w_{\eta}\right) M_{\eta} \preccurlyeq 0$, because for any $v$

$$
\left\langle v, M_{\eta} v\right\rangle_{k}=v^{\top} \operatorname{diag}\left(w_{k}\right) M_{\eta} v=v^{\top} \operatorname{diag}\left(w_{\eta}\right) M_{\eta} v,
$$

where $w_{k}$ is the vector of $w$ values on $X(k)$ and for the last equality we used that $w_{k}$ is the same as $w_{\eta}$ on all $\tau \supset \eta$.

Thus, it suffices to prove that $A_{\eta} \preccurlyeq \frac{w_{\eta} w_{\eta}^{\top}}{w(\eta)}$. We view $A_{\eta}$ as the weighted adjacency matrix of the 1-skeleton (which we recall is a graph) of the link $X_{\eta}$. Again, note that $A_{\eta}$ is symmetric. First, we claim it suffices to show that $\tilde{P}_{\eta, 1}^{\wedge}=\operatorname{diag}\left(w_{\eta}\right)^{-1} A_{\eta}$ gives the corresponding non-lazy simple random walk matrix. Since $(X, w)$ is a 0-local spectral expander, $\tilde{P}_{\eta, 1}^{\wedge}$ has at most one positive eigenvalue, whence $A_{\eta}=\operatorname{diag}\left(w_{\eta}\right) \cdot \tilde{P}_{\eta, 1}^{\wedge}$ has at most one positive eigenvalue by Lemma 2.5. Since $w(\eta)=\sum_{\tau \in X(k): \tau \supset \eta} w(\tau), A_{\eta} \preccurlyeq \frac{w_{\eta} w_{\eta}^{\top}}{w(\eta)}$ then follows immediately by Lemma 2.6 .

To see that $\tilde{P}_{\eta, 1}^{\wedge}=\operatorname{diag}\left(w_{\eta}\right)^{-1} A_{\eta}$, we verify that $w_{\eta}$ are the row sums of $A_{\eta}$. Observe that for every $\tau \in X(k)$ satisfying $\tau \supset \eta$,

$$
\begin{aligned}
\sum_{\sigma \in X(k)} A_{\eta}(\tau, \sigma) & =\sum_{\sigma \in X(k): \sigma \cup \tau \in X(k+1), \sigma \cap \tau=\eta} w(\tau \cup \sigma) \\
& =\sum_{\varphi \in X(k+1): \varphi \supset \tau} w(\varphi)=w(\tau)
\end{aligned}
$$

where we use that for each $\varphi \in X(k+1)$ satisfying $\varphi \supset \tau$, there is a unique $\sigma \in X(k)$ satisfying $\sigma \cap \tau=\eta$ and $\sigma \cup \tau=\varphi$ (this $\sigma$ is given by $\eta \cup(\varphi \backslash \tau))$. The last step uses the balancing condition.

Proof of Theorem 3.3. We go by induction on $k$. The case $k=$ 0 is trivial, as $P_{0}^{\wedge}$ is $1 \times 1$. When $k=1$, we have $P_{1}^{\wedge}=\frac{1}{2}\left(\tilde{P}_{1}^{\wedge}+I\right)$. As $(X, w)$ is a 0 -local spectral expander, $\tilde{P}_{1}^{\wedge}$ has exactly one positive eigenvalue, with value 1 . Hence, $P_{1}^{\wedge}$ has eigenvalue 1 with multiplicity 1 . All other eigenvalues of $P_{1}^{\wedge}$ are less than or equal to $1 / 2$, of which, there are $|X(1)|-1$ many. Thus, the base case holds.

Assume the claim holds for some $d-1>k \geqslant 0$. Recall by Lemma 3.1, $P_{k+1}^{\vee}$ has the same non-zero eigenvalues as $P_{k}^{\wedge}$. By Lemma 3.5,

$$
P_{k+1}^{\wedge} \preccurlyeq_{k+1} \frac{k+1}{k+2} P_{k+1}^{\vee}+\frac{1}{k+2} I
$$

For $-1 \leqslant i \leqslant k, P_{k}^{\wedge}$ as at most $|X(i)|$ eigenvalues $>1-\frac{i+1}{k+1}$. Hence, $P_{k+1}^{\wedge}$ has at most $|X(i)|$ eigenvalues $>\frac{k+1}{k+2} \cdot\left(1-\frac{i+1}{k+1}\right)+\frac{1}{k+2}=$ $1-\frac{i+1}{k+2}$. For $i=k+1$, we trivially have that $P_{k+1}^{\wedge}$ has at most $|X(k+1)|$ eigenvalues $>0$, as $P_{k+1}^{\wedge}$ is $|X(k+1)| \times|X(k+1)|$.

\section{FROM STRONGLY LOG-CONCAVE POLYNOMIALS TO LOCAL SPECTRAL EXPANDERS}

In this section, we prove Theorem 1.1. Let $p=\sum_{S} c_{S} x^{S}$ be a $d$ homogeneous multiaffine strongly log-concave polynomial with nonnegative coefficients. We can construct a pure $d$-dimensional complex $X^{p}$ from $p$ as follows: For every term $c_{S} x^{S}$ of $p$ we include the $d$-dimensional simplex $S$ with weight

$$
w(S)=c_{S} .
$$

Note that since $p$ has nonnegative coefficients, the above weight function is nonnegative. We turn $X^{p}$ into a simplicial complex by including all subsets of the $d$-dimensional simplices and weighting each lower dimensional simplex inductively according to Eq. (1).

Proposition 4.1. Let $p \in \mathbb{R}\left[x_{1}, \ldots, x_{n}\right]$ be a multiaffine homogeneous polynomial with nonnegative coefficients. If $p$ is strongly logconcave then $\left(X^{p}, w\right)$ is a 0 -local-spectral-expander, where $w(S)=c_{S}$ for every maximal face $S \in X^{p}$.

The converse of the above statement also holds true and we will discuss it in the next section. We build up to the proof of Proposition 4.1 and thereby the proof of Theorem 1.1.

We now fix a multiaffine $d$-homogeneous strongly log-concave polynomial $p$ with nonnegative coefficients. Fix a simplex $\tau \in X^{p}(k)$ and let $p_{\tau}=\left(\prod_{i \in \tau} \partial_{i}\right) p$. Note that $p_{\tau}$ is $(d-k)$-homogeneous.

Lemma 4.2. For any $0 \leqslant k \leqslant d$, and any simplex $\tau \in X^{p}(k)$, $w(\tau)=(d-k) ! \cdot p_{\tau}(\mathbb{1})$.

Proof Sketch. The claim follows by induction on $d-k$, using the fact that the weights $w(\tau)$ are balanced for the inductive step. The full proof is given in the full version of the paper [Ana+18a].

Recall that $\tilde{P}_{\tau, 1}^{\wedge}$ is the transition probability matrix of the nonlazy random walk on the 1 -skeleton of the link $X_{\tau}^{p}$. To prove the Proposition 4.1 it is enough to show that $\lambda_{2}\left(\tilde{P}_{\tau, 1}^{\wedge}\right) \leqslant 0$, i.e. that $\tilde{P}_{\tau, 1}^{\wedge}$ has at most one positive eigenvalue.

Proof of Proposition 4.1. Since $p$ is strongly log-concave, the Hessian $\nabla^{2} p_{\tau}(\mathbb{1})$ has at most one positive eigenvalue. Let

$$
\tilde{\nabla}^{2} p_{\tau}=\frac{1}{d-k-1} \operatorname{diag}\left(\nabla p_{\tau}(\mathbb{1})\right)^{-1} \nabla^{2} p_{\tau}(\mathbb{1}) .
$$

We claim that

$$
\tilde{\nabla}^{2} p_{\tau}=\tilde{P}_{\tau, 1}^{\wedge}
$$

To see this, note that by Eq. (4), for $i, j \in X_{\tau}^{p}$,

$$
\tilde{P}_{\tau, 1}^{\wedge}(i, j)=\frac{w_{\tau}(\{i, j\})}{w_{\tau}(\{i\})}=\frac{w(\tau \cup\{i, j\})}{w(\tau \cup\{i\})} .
$$

On the other hand, by Eq. (7),

$$
\left(\tilde{\nabla}^{2} p_{\tau}\right)(i, j)=\frac{\left(\partial_{i} \partial_{j} p_{\tau}\right)(\mathbb{1})}{(d-k-1) \cdot\left(\partial_{i} p_{\tau}\right)(\mathbb{1})} .
$$


The above two are equal by Lemma 4.2, which proves Eq. (8).

Since $p$ has nonnegative coefficients, the vector $\nabla p_{\tau}(\mathbb{1})$ has nonnegative entries, which implies $\operatorname{diag}\left(\nabla p_{\tau}(\mathbb{1})\right) \succcurlyeq 0$. Since $\nabla^{2} p_{\tau}(\mathbb{1})$ has at most one positive eigenvalue, $\tilde{\nabla}^{2} p_{\tau}(\mathbb{1})$ has at most one positive eigenvalue by Lemma 2.5 as desired. Therefore by Eq. (8), $\tilde{\nabla}^{2} p_{\tau}=\tilde{P}_{\tau, 1}^{\wedge}$ has at most one positive eigenvalue and $\lambda_{2}\left(\tilde{P}_{\tau, 1}^{\wedge}\right) \leqslant$ 0 .

With this we are ready to prove our main theorem.

Proof of Theorem 1.1. Let $\mu$ be a $d$-homogeneous strongly logconcave distribution and let $P_{\mu}$ be the transition probability matrix of the chain $\mathcal{M}_{\mu}$. By Theorem 2.7 it is enough to show that $\lambda^{*}\left(P_{\mu}\right) \leqslant$ $1-1 / d$. Observe that the chain $\mathcal{M}_{\mu}$ is exactly the same as the chain $P_{d}^{\vee}$ for the simplicial complex $X^{g_{\mu}}$ defined above. Therefore, $\lambda^{*}\left(P_{\mu}\right)=\lambda^{*}\left(P_{d}^{\vee}\right)=\lambda^{*}\left(P_{d-1}^{\wedge}\right)$, where the last equality follows by Lemma 3.1. Since $g_{\mu}$ is strongly log-concave, by Proposition 4.1, $X^{g_{\mu}}$ is 0-local-spectra-expander. Therefore, by Theorem 3.3,

$$
\lambda^{*}\left(P_{d-1}^{\wedge}\right) \leqslant 1-\frac{1}{(d-1)+1}=1-\frac{1}{d}
$$

as desired.

\subsection{Bases Exchange Walk}

In this part we prove Theorem 1.5. Fix a rank $r$ matroid $M=([n], \mathcal{I})$, let $\mu$ denote the uniform distribution on the bases of $M$, and consider the simplicial complex $X^{g_{\mu}}$. As before, recall that $P_{r}^{\vee}$ is the transition matrix for the Markov chain $\mathcal{M}_{\mu}$ defined in the introduction. As discussed in Section 2.2, each reversible Markov chain is equivalent to a random walk in a (weighted) undirected graph. Let $H_{M}$ be the graph corresponding to $P_{r}^{\vee}$. Then the vertices of $H_{M}$ correspond to bases of $M$, and the weight of an edge between two bases $\tau, \tau^{\prime}$ is,

$$
P_{r}^{\vee}\left(\tau, \tau^{\prime}\right)=P_{r}^{\vee}\left(\tau^{\prime}, \tau\right)
$$

The symmetry of $P_{r}^{\vee}$ follows by the fact that $P_{r}^{\vee}$ is reversible and $w(\tau)=w\left(\tau^{\prime}\right)=1$. Observe that $P_{r}^{\vee}$ is the adjacency matrix of $H_{M}$. Furthermore, note that by Theorem 6.1 (proof in Section 5), $g_{\mu}$ is a strongly log-concave polynomial and hence, $X^{g_{\mu}}$ is a 0 -local spectral expander by Proposition 4.1. By Theorem 3.3, $\lambda_{2}\left(P_{r}^{\vee}\right) \leqslant$ $1-\frac{1}{r}$. Note that the weighted degree of each vertex of $H_{M}$ is 1 since $P_{r}^{\vee}$ is a stochastic matrix. Therefore, by Cheeger's inequality, Theorem 2.8,

$$
\operatorname{cond}\left(H_{M}\right) \geqslant \frac{1-\lambda_{2}\left(P_{r}^{\vee}\right)}{2} \geqslant \frac{1-(1-1 / r)}{2}=\frac{1}{2 r} .
$$

Let $G_{M}=(\mathcal{B}, E)$ be the (unweighted) bases exchange graph associated to $M$ as defined in the introduction. It follows that $G_{M}$ is the unweighted base graph of $H_{M}$. Fix a nonempty set $S \subset \mathcal{B}$ of bases such that $|S| \leqslant|\mathcal{B}| / 2$. We need to show that the expansion $\mathrm{h}(S)$ is $\geqslant 1$. Note that since the weighted degree of each vertex of $H_{M}$ is $1, \operatorname{vol}_{H}(S)=|S|$. It follows that

$$
\begin{aligned}
\operatorname{cond}\left(H_{M}\right) & \leqslant \operatorname{cond}(S)=\frac{\sum_{\tau, \tau^{\prime}: \tau \in S, \tau^{\prime} \notin S} P_{r}^{\vee}\left(\tau, \tau^{\prime}\right)}{|S|} \\
& \leqslant \frac{\sum_{\tau, \tau^{\prime}: \tau \in S, \tau^{\prime} \notin S} \frac{1}{2 r}}{|S|}=\frac{\frac{1}{2 r}|E(S, \bar{S})|}{|S|}=\frac{\mathrm{h}(S)}{2 r}
\end{aligned}
$$

The second inequality follows by the following fact. Putting the above together with Eq. (9), implies $\mathrm{h}(S) \geqslant 1$ as desired. This completes the proof of Theorem 1.5.

Fact 4.3. For any pair of bases, $\tau, \tau^{\prime} \in X^{g_{\mu}}(r)$,

$$
P_{r}^{\vee}\left(\tau, \tau^{\prime}\right) \leqslant \frac{1}{2 r} .
$$

Proof. Suppose $P_{r}^{\vee}\left(\tau, \tau^{\prime}\right)>0$. This means that $\left|\tau \cap \tau^{\prime}\right|=r-1$. Therefore,

$$
\begin{aligned}
& w\left(\tau \cap \tau^{\prime}\right) \geqslant w(\tau)+w\left(\tau^{\prime}\right)=2 . \\
& \text { So, } P_{r}^{\vee}\left(\tau, \tau^{\prime}\right)=\frac{w\left(\tau^{\prime}\right)}{r w\left(\tau \cap \tau^{\prime}\right)} \leqslant \frac{1}{2 r} .
\end{aligned}
$$

\section{THE DEGREE-2 CHARACTERIZATION OF STRONG LOG-CONCAVITY}

In this part we prove Theorem 2.14 using connections with high dimensional expanders. Theorem 2.14 can be seen as the converse of Proposition 4.1. In fact, the following statement was proved by Oppenheim [Opp18].

Theorem 5.1 ([Opp18]). Let $(X, w)$ be a pure weighted simplicial complex of dimension-d such that:

(1) for all $\tau \in X(k)$, for $0 \leqslant k \leqslant d-2$, the 1-skeleton of $X_{\tau}$ is a connected (weighted) graph, and

(2) for any $\tau \in X(d-2), \lambda_{2}\left(\tilde{P}_{\tau, 1}^{\wedge}\right) \leqslant 0$.

Then $(X, w)$ is a weighted 0 -local-spectral-expander.

Our proof of Theorem 2.14 can be seen as a translation of Oppenheim's result into the language of polynomials. ${ }^{2}$ We actually prove a slightly stronger statement, in the sense that a direct translation of Theorem 5.1 corresponds to testing strong log-concavity of a multiaffine homogeneous polynomial. In this case, each derivative $\partial_{\tau} p$ corresponds to taking the link of $\tau$ in $X^{p}$. Indecomposability of each derivative then corresponds to connectivity of the 1-skeleton of the corresponding link, and log-concavity of quadratics corresponds to $\lambda_{2}\left(\tilde{P}_{\tau, 1}^{\wedge}\right) \leqslant 0$.

Proof of Theorem 2.14. We proceed by induction on the degree of $p$. If the degree of $p$ is at most 2, the claim obviously holds. So, suppose $d \geqslant 3$. For any $1 \leqslant i \leqslant n$, let $p_{i}$ denote $\partial_{i} p$. By induction, we can assume that for all $i, p_{i}$ is strongly log-concave (at $1)$.

First, by Eq. (3), $\nabla^{2} p(\mathbb{1})=\frac{1}{d-2} \sum_{i=1}^{n} \nabla^{2} p_{i}(\mathbb{1})$. By induction and Proposition 2.11, each matrix $\nabla^{2} p_{i}(\mathbb{1})$ has at most one positive eigenvalue.

Instead, we work with the normalized Hessian matrix, $\tilde{\nabla}^{2} p=$ $\frac{1}{d-1} \operatorname{diag}(\nabla p(\mathbb{1}))^{-1} \nabla^{2} p(\mathbb{1})$ as defined in Eq. (7). Since the normalized Hessian matrix is stochastic, its top eigenvector is the all-ones vector. When working with the normalized Hessian we need to use the correct inner product operators. For a $d$-homogeneous polynomial $p$ with nonnegative coefficients and $d>1$, and vectors $\phi, \psi \in \mathbb{R}^{n}$, define

$$
\langle\phi, \psi\rangle_{p}=(d-1) \sum_{j=1}^{n} \phi(j) \psi(j)\left(\partial_{j} p(\mathbb{1})\right),
$$

${ }^{2}$ In a companion paper, we will give an alternative proof based purely on elementary calculus and linear algebra. 
which gives the norm $\|\phi\|_{p}^{2}=\langle\phi, \phi\rangle_{p}$. The following identity is immediate:

$$
\left\langle\phi,\left(\tilde{\nabla}^{2} p\right) \psi\right\rangle_{p}=\left\langle\phi, \nabla^{2} p(\mathbb{1}) \psi\right\rangle=\left\langle\left(\tilde{\nabla}^{2} p\right) \phi, \psi\right\rangle_{p}
$$

In particular, $\tilde{\nabla}^{2} p$ is self-adjoint with respect to $\langle\cdot, \cdot\rangle_{p}$. Furthermore, by Eq. (3),

$$
\begin{aligned}
\left\langle\phi,\left(\tilde{\nabla}^{2} p\right) \psi\right\rangle_{p} & =\left\langle\phi, \nabla^{2} p(\mathbb{1}) \psi\right\rangle=\frac{1}{d-2} \sum_{k=1}^{n}\left\langle\phi, \nabla^{2} p_{k}(\mathbb{1}) \psi\right\rangle \\
& =\frac{1}{d-2} \sum_{k=1}^{n}\left\langle\phi, \tilde{\nabla}^{2} p_{k} \psi\right\rangle_{p_{k}}
\end{aligned}
$$

We highlight that $\nabla^{2} p(\mathbb{1})$ may be viewed as the weighted adjacency matrix of a graph with edge weights $\partial_{i} \partial_{j} p(\mathbb{1})$. Thus, our normalized Hessian may be viewed as the associated random walk matrix, and the inner product $\langle\cdot, \cdot\rangle_{p}$ may be viewed as a change of basis, which converts the random walk matrix into the normalized adjacency matrix.

Let $\mu$ be an eigenvalue of $\tilde{\nabla}^{2} p$ with eigenvector $\phi$. We prove that $\mu \leqslant \mu^{2}$. We claim that this is enough for the induction step: First, since $\tilde{\nabla}^{2} p$ is stochastic $\mu \leqslant 1$. Therefore, we either $\mu=1$ or $\mu \leqslant 0$. So, to prove that $\tilde{\nabla}^{2} p$ has (exactly) one positive eigenvalue, it is enough to show that $\lambda_{2}\left(\tilde{\nabla}^{2} p\right)<1$. But, since $p$ is indecomposable, the underlying (weighted) graph of $\tilde{\nabla}^{2} p$ is connected, so by Corollary $2.9, \lambda_{2}\left(\tilde{\nabla}^{2} p\right)<1$.

It remains to prove that $\mu \leqslant \mu^{2}$. From Eq. (10),

$$
\mu\|\phi\|_{p}^{2}=\left\langle\phi,\left(\tilde{\nabla}^{2} p\right) \phi\right\rangle_{p}=\frac{1}{d-2} \sum_{k=1}^{n}\left\langle\phi,\left(\tilde{\nabla}^{2} p_{k}\right) \phi\right\rangle_{p_{k}}
$$

Decomposing $\phi$ orthogonally along $\mathbb{1}$, write $\phi=\phi_{k}^{\perp \mathbb{1}}+\phi_{k}^{\mathbb{1}}$ where $\phi_{k}^{\mathbb{1}}=\frac{\langle\phi, \mathbb{1}\rangle_{p_{k}}}{\|\mathbb{1}\|_{p_{k}}^{2}} \mathbb{1}$ and $\phi_{k}^{\perp \mathbb{1}}$ is orthogonal to $\mathbb{1}$, i.e., $\left\langle\phi_{k}^{\perp \mathbb{1}}, \mathbb{1}\right\rangle_{p_{k}}=0$. It follows that

$$
\left\langle\phi_{k}^{\perp \mathbb{1}},\left(\tilde{\nabla}^{2} p_{k}\right) \phi_{k}^{\perp \mathbb{1}}\right\rangle_{p_{k}} \leqslant 0 .
$$

This is because $\tilde{\nabla}^{2} p_{k}$ has exactly one positive eigenvalue with corresponding eigenvector of $\mathbb{1}$. Therefore,

$$
\begin{aligned}
\mu\|\phi\|_{p}^{2} & \leqslant \frac{1}{d-2} \sum_{k=1}^{n}\left\langle\phi_{k}^{\mathbb{1}},\left(\tilde{\nabla}^{2} p_{k}\right) \phi_{k}^{\mathbb{1}}\right\rangle_{p_{k}} \\
& =\frac{1}{d-2} \sum_{k=1}^{n}\left\langle\phi_{k}^{\mathbb{1}}, \phi_{k}^{\mathbb{1}}\right\rangle_{p_{k}}=\frac{1}{d-2} \sum_{k=1}^{n} \frac{\langle\phi, \mathbb{1}\rangle_{p_{k}}^{2}}{\langle\mathbb{1}, \mathbb{1}\rangle_{p_{k}}}
\end{aligned}
$$

Next, we rewrite the numerator and denominator of each ratio in the righthand side. We have

$$
\langle\mathbb{1}, \mathbb{1}\rangle_{p_{k}}=(d-2) \sum_{i=1}^{n}\left(\partial_{i} p_{k}(\mathbb{1})\right)=(d-2)(d-1) \cdot p_{k}(\mathbb{1})
$$

where we used Eq. (2) for polynomial $p_{k}$. Furthermore,

$$
\begin{aligned}
\langle\phi, \mathbb{1}\rangle_{p_{k}} & =(d-2) \sum_{i=1}^{n} \phi(i)\left(\partial_{i} p_{k}(\mathbb{1})\right) \\
& =(d-2) \cdot\left(\left(\nabla^{2} p(\mathbb{1})\right) \phi\right)(k)
\end{aligned}
$$

So, putting the above identities together, we obtain

$$
\begin{aligned}
\frac{\langle\phi, \mathbb{1}\rangle_{p_{k}}}{\langle\mathbb{1}, \mathbb{1}\rangle_{p_{k}}} & =\frac{1}{(d-1) \cdot p_{k}}\left(\left(\nabla^{2} p(\mathbb{1})\right) \phi\right)(k) \\
& =\left(\left(\tilde{\nabla}^{2} p(\mathbb{1})\right) \phi\right)(k)=\mu \cdot \phi(k)
\end{aligned}
$$

where the last identity we crucially used $\phi$ is the eigenvector of $\tilde{\nabla}^{2} p$ corresponding to $\mu$. Plugging into Eq. (12) we get

$$
\begin{aligned}
\mu\|\phi\|_{p}^{2} & \leqslant \frac{1}{d-2} \sum_{k=1}^{n} \frac{\langle\phi, \mathbb{1}\rangle_{p_{k}}^{2}}{\langle\mathbb{1}, \mathbb{1}\rangle_{p_{k}}}=\frac{\mu}{d-2} \sum_{k=1}^{n} \phi(k)\langle\phi, \mathbb{1}\rangle_{p_{k}} \\
& =\mu \sum_{k=1}^{n} \phi(k)\left(\left(\nabla^{2} p(\mathbb{1})\right) \phi\right)(k)=\mu\left\langle\phi,\left(\nabla^{2} p(\mathbb{1})\right) \phi\right\rangle \\
& =\mu\left\langle\phi,\left(\tilde{\nabla}^{2} p\right) \phi\right\rangle_{p}=\mu^{2}\|\phi\|_{p}^{2} .
\end{aligned}
$$

The second equality uses Eq. (13) and the last equality uses definition of $\phi$. So, $\mu \leqslant \mu^{2}$ as desired.

\section{AN ALTERNATIVE PROOF OF STRONG LOG-CONCAVITY OF THE BASES GENERATING POLYNOMIAL}

Theorem 6.1. Let $M=([n], \mathcal{I})$ be a matroid of rank $r$. Then, for every choice of "external field" $\lambda=\left(\lambda_{1}, \ldots, \lambda_{n}\right) \in \mathbb{R}_{>0}^{n}$, its (weighted) bases generating polynomial

$$
g_{M}\left(x_{1}, \ldots, x_{n}\right)=\sum_{B \text { basis }} \lambda^{B} x^{B}
$$

is strongly log-concave (at $\mathbb{1}$ ).

Proof. We verify the indecomposability and log-concavity conditions of Theorem 2.14. If $i_{1}, \ldots, i_{k}$ contains duplicate elements, then multiaffine-ness of $g_{M}$ forces $\partial_{i_{1}} \cdots \partial_{i_{k}} g_{M}$ to be identically zero. For any subset $S=\left\{i_{1}, \ldots, i_{k}\right\}$, we use $\partial_{S}$ as a shorthand notation for $\partial_{i_{1}} \cdots \partial_{i_{k}}$. If $S$ is not independent, then no basis contains $S$, and again, $\partial_{S} g_{M}=0$ identically. Hence, we assume $S \in \mathcal{I}$.

We first argue that $\partial_{S} g_{M}$ is indecomposable. Observe that $\partial_{S} g_{M}$ equals the weighted basis generating polynomial $g_{M / S}$ of the contraction $M / S$. As $M / S$ is a matroid of rank $\geqslant 2$, applying the exchange property immediately tells us that $g_{M / S}$ is indecomposable.

Now, we verify log-concavity of all quadratics. Assume $S \in \mathcal{I}$ and $|S|=r-2$. As $S$ has rank- $(r-2), M / S$ has rank two. In particular, $\partial_{S} g_{M}=g_{M / S}$ is quadratic and $\nabla^{2} g_{M / S}$ has entries

$$
\left(\nabla^{2} g_{M / S}\right)_{i j}= \begin{cases}\lambda_{i} \lambda_{j}, & \text { if }\{i, j\} \text { is independent in } M / S \\ 0, & \text { otherwise. }\end{cases}
$$

We need to prove that the above matrix has at most one positive eigenvalue.

For any set $T \subseteq[n]$, let $\lambda_{T}$ denote the vector with $i$ th entry $\lambda_{i}$ for $i \in T$ and 0 for $i \notin T$. The matroid partition property tells us that we can partition the non-loops of $M / S$ into blocks $B=B_{1} \cup \cdots \cup B_{k}$. Then

$$
\nabla^{2} g_{M / S}=\lambda_{B} \lambda_{B}^{\top}-\sum_{i=1}^{k} \lambda_{B_{i}} \lambda_{B_{i}}^{\top} \preccurlyeq \lambda_{B} \lambda_{B}^{\top}
$$

This proves that $\nabla^{2} \partial_{S} g_{M}$ has at most one positive eigenvalue and thus $\partial_{S} g_{M}$ is log-concave. 
The proof of Theorem 1.6 is very similar, and is given in the full version of the paper $[$ Ana+18a]. We also defer the proof of Theorem 1.7 to $[$ Ana+18a].

\section{ACKNOWLEDGMENTS}

Part of this work was started while the first and last authors were visiting the Simons Institute for the Theory of Computing. It was partially supported by the DIMACS/Simons Collaboration on Bridging Continuous and Discrete Optimization through NSF grant CCF1740425. Shayan Oveis Gharan and Kuikui Liu are supported by the NSF grant CCF-1552097 and ONR-YIP grant N00014-17-1-2429. Cynthia Vinzant was partially supported by the National Science Foundation grant DMS-1620014.

We thank Lap Chi Lau, Mark Jerrum and Alan Frieze for helpful comments on an earlier version of this manuscript.

\section{REFERENCES}

[AHK18] Karim Adiprasito, June Huh, and Eric Katz. "Hodge theory for combinatorial geometries". In: Annals of Mathematics 188.2 (2018), pp. 381452.

[Alo86] N Alon. "Eigenvalues and expanders". In: Combinatorica 6 (2 Jan. 1986), pp. 83-96. ISSN: 0209-9683.

[AM85] N. Alon and V. Milman. "Isoperimetric inequalities for graphs, and superconcentrators". In: fournal of Combinatorial Theory, Series B 38.1 (Feb. 1985), pp. 73-88.

[Ana+18a] Nima Anari, Kuikui Liu, Shayan Oveis Gharan, and Cynthia Vinzant "Log-Concave Polynomials II: High-Dimensional Walks and an FPRAS for Counting Bases of a Matroid”. In: arXiv preprint arXiv:1811.01816 (2018).

[Ana+18b] Nima Anari, Kuikui Liu, Shayan Oveis Gharan, and Cynthia Vinzant "Log-Concave Polynomials III: Mason's Ultra-Log-Concavity Conjecture for Independent Sets of Matroids". In: arXiv preprint arXiv:1811.01600 (2018).

[AOR16] Nima Anari, Shayan Oveis Gharan, and Alireza Rezaei. "Monte Carlo Markov Chain Algorithms for Sampling Strongly Rayleigh Distributions and Determinantal Point Processes". In: COLT. 2016, pp. 103-115.

[AOV18] Nima Anari, Shayan Oveis Gharan, and Cynthia Vinzant. "Log-concave polynomials, entropy, and a deterministic approximation algorithm for counting bases of matroids". In: FOCS. to appear. 2018

[BH18] Petter Brändén and June Huh. "Hodge-Riemann relations for Potts model partition functions". In: arXiv preprint arXiv:1811.01696 (2018).

[BH19] Petter Brändén and June Huh. "Lorentzian polynomials". In: arXiv preprint arXiv:1902.03719 (2019).

[BS07] Alexander Barvinok and Alex Samorodnitsky. "Random weighting, asymptotic counting, and inverse isoperimetry". In: Israel fournal of Mathematics 158.1 (Mar. 2007), pp. 159-191. ISSN: 1565-8511.

[BŚ97] W. Ballmann and J. Światkowski. "On L2-cohomology and property (T) for automorphism groups of polyhedral cell complexes”. In: Geom. Funct. Anal. 7.4 (1997), pp. 615-645.

[Clo10] Brian D. Cloteaux. "Approximating the Number of Bases for Almost All Matroids". In: Congressus Numerantium 202 (2010), pp. 149-154.

[CTY15] Emma Cohen, Prasad Tetali, and Damir Yeliussizov. "Lattice path matroids: negative correlation and fast mixing”. In: arXiv preprint arXiv:1505.06710 (2015).

[DK17] I. Dinur and T. Kaufman. "High Dimensional Expanders Imply Agreement Expanders". In: FOCS. 2017, pp. 974-985

[DS91] Persi Diaconis and Daniel Stroock. "Geometric bounds for eigenvalues of Markov chains". In: The Annals of Applied Probability (1991), pp. 3661.

[FK72] Cornelis Marius Fortuin and Pieter Willem Kasteleyn. "On the randomcluster model: I. Introduction and relation to other models". In: Physica 57 (4 1972), pp. 536-564.

[FM92] Tomás Feder and Milena Mihail. "Balanced matroids". In: STOC. 1992 pp. 26-38.

[For71] Cornelis Marius Fortuin. "On the random-cluster model”. PhD thesis. Leiden University, 1971

[For72a] Cornelis Marius Fortuin. "On the random-cluster model: II. The percolation model”. In: Physica 58 (3 1972), pp. 393-418.
[For72b] Cornelis Marius Fortuin. "On the random-cluster model: III. The simple random-cluster model". In: Physica 59 (4 1972), pp. 545-570.
Anna Gambin. "On approximating the number of bases of exchange preserving matroids". In: International Symposium on Mathematical Foundations of Computer Science. Springer. 1999, pp. 332-342.

[Gar73] H. Garland. "p-adic curvature and the cohomology of discrete subgroups of p-adic groups". In: Annals of Mathematics 97.3 (1973), pp. 375-423.

[GJ08] L. A. Goldberg and M. Jerrum. "Inapproximability of the Tutte polynomial”. In: Inform. and Comput 206.7 (2008), pp. 908-929.

[GJ12a] L. A. Goldberg and M. Jerrum. "Approximating the partition function of the ferromagnetic Potts model". In: f. ACM 59.5 (2012), pp. 1-31.

[GJ12b] L. A. Goldberg and M. Jerrum. "Inapproximability of the Tutte polynomial of a planar graph”. In: Computational Complexity 21 (4 2012), pp. 605-642.

[GJ13] L. A. Goldberg and M. Jerrum. "Approximating the Tutte polynomial of a binary matroid and other related combinatorial polynomials". In: Journal of Computer and System Sciences 79 (1 2013), pp. 68-78.

[GJ14] L. A. Goldberg and M. Jerrum. "The complexity of computing the sign of the Tutte polynomial". In: SIAM f. Comput. 43 (2014), pp. 1921-1952.

[GJ17] Heng Guo and Mark Jerrum. "Random cluster dynamics for the Ising

[GJ17] Heng Guo and Mark Jerrum. "Random cluster dynamics for the Ising

[GJ18a] Heng Guo and Mark Jerrum. "A Polynomial-Time Approximation Algorithm for All-Terminal Network Reliability”. In: ICALP. 2018, 68:168:12.

[GJ18b] Heng Guo and Mark Jerrum. "Approximately counting bases of bicircular matroids". 2018.

[Gri09] G. R Grimmett. The Random-Cluster Model. Berlin: Springer-Verlag, 2009.

[Gur09] Leonid Gurvits. "A polynomial-time algorithm to approximate the mixed volume within a simply exponential factor". In: Discrete \& Computational Geometry 41.4 (2009), pp. 533-555.

[Gur10] Leonid Gurvits. "On multivariate Newton-like inequalities". In: Advances in Combinatorial Mathematics. Berlin, Heidelberg: Springer Berlin Heidelberg, 2010, pp. 61-78.

[HJ13] Roger A Horn and Charles R Johnson. Matrix analysis. 2nd ed. Cambridge university press, 2013.

[HW16] June Huh and Botong Wang. "Enumeration of points, lines, planes, etc". In: arXiv preprint arXiv:1609.05484 (2016).

[Jer+04] Mark Jerrum, Jung-Bae Son, Prasad Tetali, and Eric Vigoda. "Elementary bounds on Poincaré and log-Sobolev constants for decomposable Markov chains". In: Annals of Applied Probability (2004), pp. 1741-1765.

[Jer06] Mark Jerrum. "Two Remarks Concerning Balanced Matroids". In: Combinatorica 26.6 (2006), pp. 733-742.

[JS02] Mark Jerrum and Jung Bae Son. "Spectral gap and log-Sobolev constant for balanced matroids". In: FOCS. 2002, pp. 721-729.

[JS93] Mark Jerrum and Alistair Sinclair. "Polynomial-time approximation algorithms for the Ising model”. In: SIAM f. Comput. 22.5 (1993), pp. 10871116.

[JVV86] Mark Jerrum, Leslie Valiant, and Vijay Vazirani. "Random Generation of Combinatorial Structures from a Uniform Distribution". In: Theoretical Computer Science 43 (1986), pp. 169-188.

[JVW90] F. Jaeger, D. L. Vertigan, and D. J. A. Welsh. "On the computational complexity of the Jones and Tutte polynomials". In: Mathematical Proceedings of the Cambridge Philosophical Society 108 (1 1990), pp. 3553.

[KM17] Tali Kaufman and David Mass. "High Dimensional Random Walks and Colorful Expansion”. In: ITCS. 2017, 4:1-4:27.

[KO18] Tali Kaufman and Izhar Oppenheim. "High Order Random Walks: Beyond Spectral Gap”. In: APPROX/RANDOM. 2018, 47:1-47:17.

[KT12] Alex Kulesza and Ben Taskar. "Determinantal point processes for machine learning”. In: Foundations and Trends in Machine Learning 5(2-3) (2012), pp. 123-286.

[Lub17] Alexander Lubotzky. "High Dimensional Expanders". 2017.

[MS91] Milena Mihail and Madhu Sudan. Connectivity Properties of Matroids. Tech. rep. EECS Department, University of California, Berkeley, Dec. 1991.

[MV89] M. Mihail and U. Vazirani. "On the expansion of $0 / 1$ polytopes". In: Journal of Combinatorial Theory. B (1989).

[Opp18] Izhar Oppenheim. "Local spectral expansion approach to high dimensional expanders part I: Descent of spectral gaps". In: Discrete and Computational Geometry 59.2 (2018), pp. 293-330.

[Ver91] D.L. Vertigan. "On the computational complexity of tutte, jones, homfly and kauffman invariants". PhD thesis. University of Oxford, 1991.

[Wel94] D.J.A Welsh. "The computational complexity of knot and matroid polynomials". In: Discrete Mathematics 124 (1-3 1994), pp. 251-269. 